REVISTA

BRASILEIRA DE

ANESTESIOLOGIA

Official Publication of the Brazilian Society of Anesthesiology www.sba.com.br

ELSEVIER

\title{
ANESTESIOLOGIA
}

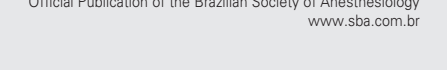

\section{Anesthetic Management of a Pediatric Patient with Leigh Syndrome}

\author{
Ismail Serhat Kocamanoglu* ${ }^{*}$, Esra Sarihasan ${ }^{2}$
}

1. MD; Associate Professor, Department of Anesthesia, Ondokuz Mayis School of Medicine, Samsun, Turkey.

2. MR, Department of Anesthesia, Ondokuz Mayis School of Medicine, Samsun, Turkey

Received from the Department of Anesthesia, Ondokuz Mayis School of Medicine, Samsun, Turkey

The summary of this paper was presented as a poster in the congress of the Turkish Anesthesiology Association, October 27, 2011, Antalya, Turkey.

Submitted on May 24, 2012. Approved on June 12, 2012.

Keywords:

Leigh Disease;

Anesthesia, General;

Monitoring, Physiologic;

Intensive Care.

\begin{abstract}
Background and objectives: Leigh syndrome (LS) is a rare disease caused by abnormalities of mitochondrial energy generation. The central nervous system is most frequently affected, with psychomotor underdevelopment, seizures, nystagmus, ophthalmoparesis, optic atrophy, ataxia, dystonia, or respiratory failure. Surgical and anesthetic procedures stimulate the tracheal irritability, and could exacerbate risks of aspiration, wheezing, breathing difficulties, gasping, hypoventilation, and apnea.

Case Report: We present the anesthetic management for a six-year-old boy with severe form of LS, involving repair of a femur fracture. Propofol and remifentanil were infused for general anesthesia. The patient was closely monitored during anesthesia and in the intensive care unit in the early postoperative period.

Conclusions: Close intraoperative monitoring of patients, including invasive arterial blood pressure monitoring and frequently measuring the levels of blood gases, glucose, and lactate, made this procedure run smoothly. Intensive care and breathing support for the patient with LS, under sedation with an analgesic combination during the early postoperative period, minimized the stress response due to pain after surgery.

๑ 2013 Sociedade Brasileira de Anestesiologia. Published by Elsevier Editora Ltda. All rights reserved.
\end{abstract}

\section{Introduction}

Leigh syndrome (LS) (subacute necrotizing encephalopathy) is a rare disease causing devastating neurodegenerative disorder that was first described by Denis Leigh in $1951^{1}$. LS is caused by abnormalities of mitochondrial energy generation, characterized by brain changes that are almost identical in all patients, such as focal, bilaterally symmetric lesions, particularly in the basal ganglia, thalamus, and brainstem, but with considerable clinical and genetic heterogeneity. Deficits in the respiratory chain (particularly of complexes I, II, IV, or V), of coenzyme Q, or of the pyruvate dehydrogenase complex are responsible for LS ${ }^{2}$. Clinically, it is characterized by a wide variety of abnormalities, from severe neurologic

*Corresponding author: Ondokuz Mayis Universitesi, Tip Fakultesi, Anestezi Anabilim Dali. Kurupelit 55139. Samsun, Turkey.

E-mail: serhatk@omu.edu.tr.

ISSN/\$ - see front metter @ 2013 @ 2013 Sociedade Brasileira de Anestesiologia. Published by Elsevier Editora Ltda. All rights reserved. 
problems to almost no abnormalities at all. The central nervous system is most frequently affected with psychomotor underdevelopment, seizures, nystagmus, ophthalmoparesis, optic atrophy, ataxia, dystonia, or respiratory failure ${ }^{3}$. These patients are in higher risk for surgery and anesthesia. Surgery and anesthesia stimulates the tracheal irritability, and could exacerbate risks of aspiration, wheezing, breathing difficulties, gasping, hypoventilation, and apnea ${ }^{4}$. So, anesthetic management is crucial in this rare disease.

We present the anesthetic management for a case with LS, involving repair of a femur fracture. The patient was closely monitored during anesthesia and in the intensive care unit in the early postoperative period. We also discuss the anesthetic choices available in literature.

\section{Case report}

The patient was a six-year-old boy, weighing $14 \mathrm{~kg}$ and measuring $120 \mathrm{~cm}$ in height, who required anesthetic care for surgery to repair a right femur fracture. Patients with LS suffer from mental-motor loss of development, necrotizing panencephalitis, convulsions, muscle and joint spasticity, and respiratory symptoms (episodes of apnea, increased airway secretions, and tachypnea). Two years before this operation, the patient had suffered from frequent vomiting and bronchopneumonia. For these reasons, he had had gastrostomy and fundoplication surgery performed under general anesthesia. The patient had needed oxygen and steam therapy due to increased airway secretions following the operation. His postoperative recovery was delayed and he was discharged 31 days after surgery.

The patient was being regularly fed through a gastrostomy tube and his family gave him lung physiotherapy for excess airway secretions. He also received muscle and joint physiotherapy for muscle spasms and spasticity. The patient's right femur had been broken during one of these physiotherapy sessions.

Coenzyme Q, vitamin E, clonazepam, carnitine, and baclofen were preoperative medications prescribed by the primary care physician. Although the patient was conscious during the pre-anesthetic visit, he showed no signs of cooperation or orientation. He had a gastrostomy catheter. The patient has deformities of the thoracic bones and scoliosis, and wheezing and rough lung sounds were detected during his physical examination (Figure 1). Preoperative laboratory evaluation revealed; hemoglobin 12.4 g. dL ${ }^{-1}$ (13-17), hematocrit $37.9 \%(40-50)$, a white cell count of $8,800 . \mu^{-1}$ $(4,300-10,300)$, and a platelet count of $265,000 \cdot \mu^{-1}(156,000-$ $363,000)$. Glucose was $89 \mathrm{mg}^{-\mathrm{dL}^{-1}}(70-110)$ and his serum lactate was $15 \mathrm{mg} \cdot \mathrm{dL}^{-1}(4-20)$. He had been fasting for six hours before surgery, in order to prevent aspiration. General anesthesia was preferred because of spasticity and difficulty in position, and the surgeon requested a neuromuscular blocking agent. The patient was constantly monitored after he arrived in the operation theatre. His preoperative vital signs were as follows: blood pressure 110/70 mm Hg; pulse 130 beats. $\mathrm{min}^{-1}$; and $\mathrm{SpO}_{2} 98 \%$.

After anesthesia induction with inhalation of sevoflurane in $\mathrm{O}_{2} / \mathrm{N}_{2} \mathrm{O}$ mixture, a 22 -gauge intravenous catheter was placed in the cubital vein. A $1 / 3$ saline-glucose mixture of fluid was infused as intravenous solution. Sevoflurane and $\mathrm{N}_{2} \mathrm{O}$ were stopped, and remifentanil infusion was started

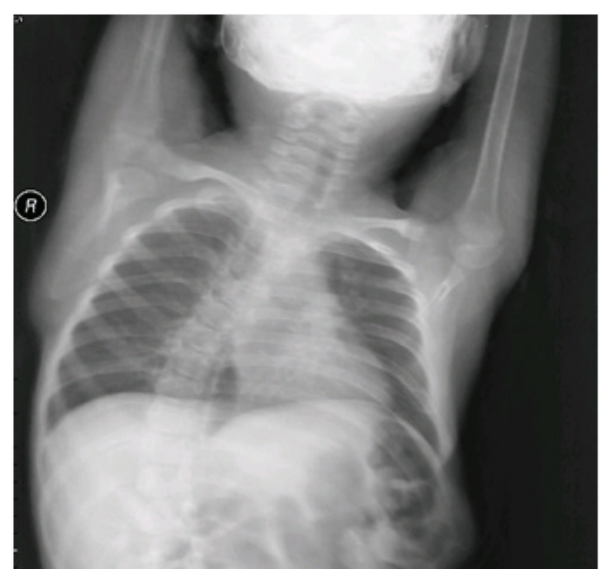

Figure 1 The patient has deformities of the thoracic bones and scoliosis.

intravenously. Propofol $20 \mathrm{mg}$ and cisatracurium $2 \mathrm{mg}$ were administered, and a cuffed tracheal tube (ID $5.0 \mathrm{~mm}$ ) was placed through the trachea. Propofol $50-150 \mu \mathrm{g} \cdot \mathrm{kg}^{-1} \cdot \mathrm{min}^{-1}$ and remifentanil 0.1-0.3 $\mu \mathrm{g} \cdot \mathrm{kg}^{-1} \cdot \mathrm{min}^{-1}$ were infused, in order to maintain anesthesia. Before the incision was made, one gram of ampicillin/sulbactam was given intravenously. A 22-G catheter was inserted into the right radial artery for blood sampling and continuous monitoring of blood pressure.

Intraoperative monitoring consisted of invasive arterial blood pressure, a three-lead electrocardiography, pulse oximetry, capnography, esophageal temperature probe, and a urethral catheter. Bronchial aspiration was performed through the tracheal tube, because of coarse lung sounds. During the anesthesia, vital signs were in the range of: blood

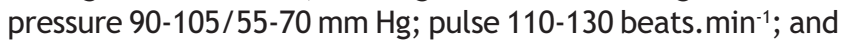
$\mathrm{SpO}_{2}$ 97-99\%. No hypotensive or hypoxic events were seen. Body temperature, values of arterial blood gases, and levels of serum lactate and glucose were all within normal range during the operation. The femur repair procedure lasted 1.5h (Figure 2). During the procedure, the patient received $200 \mathrm{~mL}$ of intravenous fluid. His total urine output was $50 \mathrm{~mL}$. While he was still intubated and receiving drug infusions, the patient was transferred to the pediatric intensive care unit, under pediatrician control.

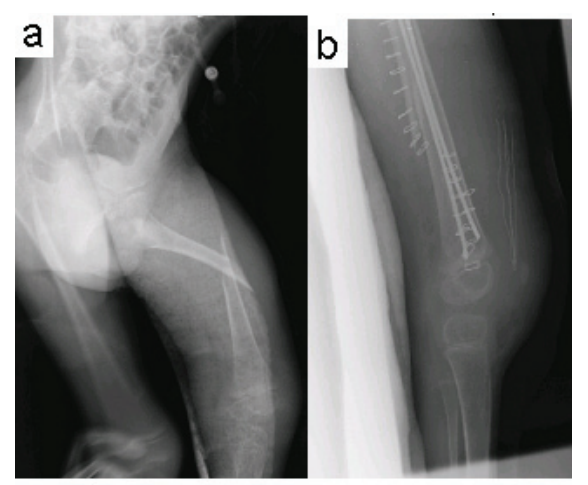

Figure 2 The views of the right femur bone of the patient before (a) and after (b) the operation. 
The patient was kept intubated for two days, receiving midazolam, fentanyl for sedation and vecuronium for muscle relaxation. The patient was extubated two days after the procedure and then transferred to orthopedic ward.

\section{Discussion}

This patient has the severe abnormalities of the most severe clinical form of LS: psychomotor underdevelopment, seizures, nystagmus, ophthalmoparesis, ataxia, respiratory failure, repeated lung infections, increase of airway secretions, and severe spasticity. About $50 \%$ of individuals affected with this disease die by age of three, most often as a result of respiratory or cardiac failure ${ }^{5}$. Although the patient had severe clinical signs, he had reached the age of six because of the attentive care and treatments he had received, and the absence of serious cardiac failure.

Spinal anesthesia would be appropriate for lower extremity surgery; as presented for muscle biopsy in a 19-month-old with LS without complication ${ }^{6}$. However, only a muscle biopsy was performed in that case, and the operation lasted just 30 minutes. In our case, the duration of the operation could not be estimated. The choice of general anesthesia was based on the surgeon's request for a neuromuscular blocking agent and the difficulty in the patient's posture due to spasticity and the fracture of his femur.

There are reports on mortality by respiratory failure for three patients after general anesthesia ${ }^{7}$. Anesthetic agents can have potentially serious negative effects in LS. Barbiturates and volatile agents can result in lactic acidosis and unfavorable metabolic manifestations ${ }^{4}$. Since vascular access was not in place when the patient came to the operating theatre, induction of anesthesia was achieved through inhalation of sevoflurane. Shenkman et al. ${ }^{4}$ also suggest that brief exposure to volatile anesthetics will not lead to metabolic deterioration. There are many reports of "propofol infusion syndrome" in children, indicating that this syndrome is not common with usual doses, but does occur when propofol is administered by infusion for more than 48 hours, and at a dose of more than $4.5 \mathrm{mg} \cdot \mathrm{kg}^{-1} \cdot \mathrm{h}^{-1}$. Most patients on short-term infusions show no clinical signs of mitochondrial disturbance ${ }^{8}$. Since succinylcholine has the risk of exaggerated rhabdomyolysis and hyperkalemia, it is not preferred in patients with myopathy. We administered cisatracurium because the surgery required muscle relaxation for a prolonged period of time and the drug's metabolism is free from the function of liver and kidney.

Lactated Ringer solution must be avoided because of its lactate content. Also, impaired glucose regulation secondary to mitochondrial dysfunction may lead to hypoglycemia during the preoperative fasting period, and dextrose containing intravenous fluids should be administered to maintain an euglycemic condition with frequent assessment of blood glucose levels during prolonged fasting ${ }^{6}$. We used glucosesaline solution for intravenous fluid therapy. To monitor metabolic changes during the operation, arterial blood gases and lactate levels were measured at frequent intervals. They were all within normal limits. Although there was no sign of infection in our case, there was evidence of increased airway secretions. Therefore, preoperative antibiotic prophylaxis was given, and bronchial aspiration was performed through the tracheal tube.

Because of the presence of preoperative reactive airways in patients who exhibit a severe picture of LS, the risk of postoperative respiratory complications is high. Anesthetic procedures stimulate the tracheal irritability, and could exacerbate the risk of pulmonary complications ${ }^{4}$. Long-term postoperative sedation is necessary to prevent the surgical stress, to reduce the risk of tracheal irritability and aspiration, and to protect wounds from the effects of the involuntary movements of patients with LS ${ }^{9}$. To ensure this was regularly monitored and that the patient received effective early treatment for pain, he was transferred to the pediatric intensive care unit under sedation. Hemodynamic, clinical and metabolic aspects of the patient were closely followed, and his tracheal tube was removed two days later.

In conclusion, selecting anesthetic agents carefully, close intraoperative monitoring of patients and frequently measuring the levels of blood gases, glucose, and lactate, are important. Intensive care and breathing support for the patient with LS, under sedation with an analgesic combination guarantee good pain control and a less stressful early postoperative period.

\section{Acknowledgement}

Published with the written consent of the patient's parent. No external funding and no competing interests declared. The authors wish to thank Dr. Alparslan Turan (Cleveland Clinic, $\mathrm{OH}, \mathrm{USA}$ ) and Dr. Gurkan Kazanci (Istanbul Fatih Sultan Mehmet Hospital) for editing the manuscript.

\section{References}

1. Leigh $D$ - Subacute necrotizing encephalomyopathy in an infant. J Neurol Neurosurg Psychiatry. 1951;14:216-221.

2. Chol M, Lebon S, Bénit P et al. - The mitochondrial DNA G13513A MELAS mutation in the NADH dehydrogenase 5 gene is a frequent cause of Leigh-like syndrome with isolated complex I deficiency. J Med Genet. 2003;40:188-191.

3. Finsterer $\mathrm{J}$ - Leigh and Leigh-like syndrome in children and adults. Pediatr Neurol. 2008;39:223-35.

4. Shenkman Z, Krichevski I, Elpeleg ON, Joseph A, Kadari A Anaesthetic management of a patient with Leigh's syndrome. Can J Anaesth. 1997;44:1091-1095.

5. Thorburn DR, Rahman S - Mitochondrial DNA-Associated Leigh Syndrome and NARP. Authors Editors In: Pagon RA, Bird TD, Dolan CR, Stephens K, editors. Source GeneReviews [Internet]. Seattle (WA): University of Washington, Seattle. http://www.ncbi.nlm.nih. gov/books/NBK1173 [1993-.2003 Oct 30 (updated 2011 May 03)].

6. Shear T, Tobias JD - Anesthetic implications of Leigh's syndrome. Paediatr Anaesth. 2004;14:792-7.

7. Grattan-Smith PJ, Shield LK, Hopkins IJ, Collins KJ - Acute respiratory failure precipitated by general anesthesia in Leigh's syndrome. J Child Neurol. 1990;5:137-141.

8. Gozal D, Goldin E, Shafran-Tikva S, Tal D, Wengrower D - Leigh syndrome: anesthetic management in complicated endoscopic procedures. Paediatr Anaesth. 2006;16:38-42.

9. Sasaki R, Nanjo K, Hirota K - Perioperative anesthetic managements for the laryngo-tracheal separation and open fundoplication in a 17-year-old patient with Leigh syndrome. Paediatr Anaesth. 2008;18:1133-1134. 